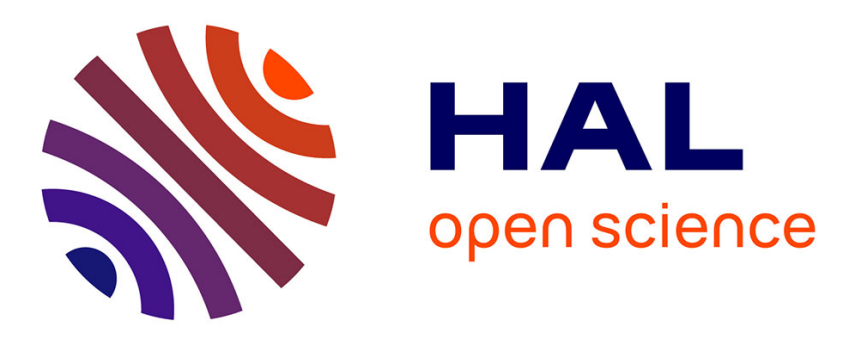

\title{
Double-Hybrid Functionals and Tailored Basis Set: Fullerene (C60) Dimer and Isomers as Test Cases
}

\author{
Eric Brémond, Ilaria Ciofini, Juan Carlos, Carlo Adamo
}

\section{To cite this version:}

Eric Brémond, Ilaria Ciofini, Juan Carlos, Carlo Adamo. Double-Hybrid Functionals and Tailored Basis Set: Fullerene (C60) Dimer and Isomers as Test Cases. Journal of Physical Chemistry A, 2019, 10.1021/acs.jpca.9b06536 . hal-02878892

\section{HAL Id: hal-02878892 \\ https://hal.science/hal-02878892}

Submitted on 10 Nov 2020

HAL is a multi-disciplinary open access archive for the deposit and dissemination of scientific research documents, whether they are published or not. The documents may come from teaching and research institutions in France or abroad, or from public or private research centers.
L'archive ouverte pluridisciplinaire HAL, est destinée au dépôt et à la diffusion de documents scientifiques de niveau recherche, publiés ou non, émanant des établissements d'enseignement et de recherche français ou étrangers, des laboratoires publics ou privés. 


\title{
Double-Hybrid Functionals and Tailored Basis Set: Fullerene $\left(\mathbf{C}_{60}\right)$ Dimer and Isomers as Test Cases
}

\author{
Éric Brémond $^{1}$, Ilaria Ciofini ${ }^{2 *}$, Juan Carlos Sancho-García ${ }^{3}$ and Carlo Adamo ${ }^{2,4^{*}}$ \\ Université de Paris, ITODYS, UMR CNRS 7086, 15 rue J.-A. de Baïf, F-75013 Paris, France; \\ Chimie ParisTech, PSL Research University, CNRS, Institut de Recherche de Chimie Paris, \\ 11, rue Pierre et Marie Curie, F-75005 Paris, France, Departamento de Química Física, \\ Universidad de Alicante, E-03080 Alicante, Spain; Institut Universitaire de France, 103 \\ Boulevard Saint Michel, F-75005 Paris, France.
}

\begin{abstract}
A computational protocol making use of Double Hybrid functionals in conjunction with a recently developed basis set tailored to reproduce non covalent interactions (hereafter named DH-SVPD) is here applied and tested for the evaluation of properties $\mathrm{C}_{60}$ fullerenes namely intermolecular interactions in the weakly bound $\mathrm{C}_{60}$ dimer and relative stabilities of $\mathrm{C}_{60}$ isomers (as described by the C60ISO and iso-C60 datasets).

The obtained results suggest that the DH-SVPD performance is very close to that obtained with empirical corrections and larger quadruple- $\zeta$ basis for the $\mathrm{C}_{60}$ dimer. In contrast, both approaches (tailored basis set and larger basis with empirical potential) do not reach the envisaged accuracy for the relative stabilities of $\mathrm{C}_{60}$ isomers.

Nevertheless, this test well underlined how the DH-SVPD basis set is able to recover the performance obtained by coupling the DH functionals with empirical dispersion corrections and larger basis set, significantly reducing the computational effort for double hybrids and thus enabling to expand their application domain to larger molecular systems.
\end{abstract}

1) Université de Paris, 2) Chimie ParisTech; 3) Universidad de Alicante ; 4) IUF

*Corresponding author: carlo.adamo@chimie-paristech.fr; ilaria.ciofini@ chimie-paristech.fr 


\section{Introduction}

Double hybrid (DH) functionals ${ }^{1}$, that are exchange-correlation functionals casting a fraction of correlation computed using a second order perturbative (PT2) approach ${ }^{2}$, represent a further step forward with respect to the global hybrids $(\mathrm{GH})$, introduced by Becke more than 20 years ago ${ }^{3}$. Indeed, modern DH schemes, firstly proposed by Truhlar ${ }^{4}$ and then popularized by Grimme ${ }^{5}$, provide enhanced performance for a number of properties, ranging from molecular structures to thermochemistry, reaction barriers, and even excited-state energies ${ }^{1}$. Contrarily to what can be expected from an error compensation between the (overbinding) PT2 and the (underbinding) DFT contributions, weak dispersion interactions are yet not very accurately reproduced by $\mathrm{DHs}^{6-9}$

An effective way to overcome this problem is to add an empirical correction based on pairwise classical potential ${ }^{10,11}$. This simple and chemically sound approach meets a large success since in such a way interaction energies of non-covalently bonded or of weakly interacting systems can be reproduced with minimal computational extra cost and high accuracy $^{12}$. Such potentials depend, of course, both on the atom types involved in the interactions and on the exchange-correlation functional considered ${ }^{13,14}$. Empirical corrections accounting for three-body interactions have been also developed, enabling to further increase the overall accuracy ${ }^{15}$.

When such empirical potentials are coupled to DHs, small errors are found on dispersionruled systems ${ }^{8}$, even if their contribution is lower than what found when these corrections are used in conjunction with more traditional functionals, such as GHs. Indeed, DHs provide a better estimation of the dispersion effects at intermediate intermolecular distances, which shows the delicate interplay between electronic effects at all ranges. ${ }^{16}$

In this paper we will focus on the behavior of two different double hybrids: the B2-PLYP and the PBE-QIDH ${ }^{17}$ functional. The first was developed in analogy to the parent B3LYP approach $^{18}$, that is fitting the (few) coefficients contained its functional form on some reference dataset ${ }^{5}$. The second, instead, has been developed choosing a number of theoretical constraints among those that the universal functional should fulfill ${ }^{17,19-21}$.

For the sake of clearness, hereafter we will denoted as non-empirical all the exchangecorrelation functionals whose internal coefficients are not determined by an error minimization procedure with respect to external reference datasets, but rather fixed using only theoretical arguments. The PBE-QIDH functional is thus ascribed to this category. By opposition, semi-empirical are those functionals whose coefficients are determined by a 
parametrization procedure, albeit their functional form could respect some theoretical constraints $^{22}$. The B2-PLYP is the most widely used DH for this category.

Potentials developed for weak interactions are, instead, empirical by their own definition, since both their form and coefficients are determined on the basis of the best agreement with respect to external reference values.

It then clearly appears how the present situation is vexing, since non-empirical DH functionals must be partnered with empirical potential in order to provide accurate results for dispersion interactions.

With this inconsistency in mind, we have recently developed a basis set, called DH-SVPD, which significantly improves the performances of DHs in reproducing interaction energies of noncovalent systems, without requiring further corrections and thus allowing to restore a full non-empirical DFT approach ${ }^{23}$. This philosophy is even more stringent, since the DH-SVPD basis set is developed using a "self-consistent" approach where only energy contributions of the monomers and dimer computed at the same DH level of theory are considered, without need of any external reference values ${ }^{24}$.

The first tests carried out on standard benchmarks, such as $\mathrm{S} 22^{25}, \mathrm{~S}_{6} 6^{26}$ and $\mathrm{L}^{27}$ dataset, suggested that the PBE-QIDH/DH-SVPD model is well suited for noncovalent interactions, providing results close (or even better) than those obtained when the same $\mathrm{DH}$ is coupled to larger basis set and empirical dispersion corrections ${ }^{23}$. They also showed the transferability of this basis set to other DHs.

Here we want firstly to verify the robustness of this approach extending our investigation to larger systems, namely the weakly bound dimer of $\mathrm{C}_{60}$. This system represents indeed a challenging playground for any method rooted in Density Functional Theory (DFT) as pointed out by a recent work $^{28}$, due to the dispersion forces which dominate the intermolecular interaction. Next, two sets concerning the relative stabilities of $\mathrm{C}_{60}$ isomers were considered ${ }^{29,30}$. Albeit composed by the same molecule, the relative stabilities of the $\mathrm{C}_{60}$ isomers seem not affected by dispersion, but their accurate evaluation at the DFT level is still troublesome, being related to an accurate description of the aromatic resonance structures. It is therefore interesting to verify the performance of our model on these two challenging sets in order to fully validate this approach beyond purely noncovalent interactions.

\section{Computational details}

Calculations were carried out with the non-empirical PBE0-DH ${ }^{31}$ and PBE-QIDH ${ }^{17}$ functionals and the semi-empirical B2-PLYP ${ }^{5}$ and DSD-PBEP86 $6^{32}$ approaches. In some cases, 
these functionals have been coupled to the empirical D3(BJ $)^{33}$ dispersion correction whose paramters are reported in Refs 34 and 35 for non-empirical DHs. Preliminary tests showed that, in contrast to our previous experience with smaller noncovalent systems (see comments in reference 21), the effect of three body-interactions, modelled by the Axilrod-Teller-Muto (ATM) potential ${ }^{15}$, were negligible for the $\mathrm{C}_{60}$ dimer. Indeed, this contribution was computed to be $0.18 \mathrm{kcal} / \mathrm{mol}$, that is about $1 \%$ of the PBE-QIDH interaction energy. Therefore, this contribution will be not considered in the following.

Note that the DH-SVPD basis set has been developed to be used in connection with any DH functional, by optimizing the diffuse functions of the small Def2-SVPD basis set ${ }^{36}$. Since this basis set has been developed considering an error compensation between Basis Set Superposition Error (BSSE) and Basis Set Incompleteness Error (BSIE), the former will be not explicitly taken into account, if not explicitly mentioned. This approach is consistent with some recent works which do not recommended BSSE corrections for small or medium basis $\operatorname{set}^{37,38}$ due to an unbalanced behavior between BSSE and BSIE. Moreover, the so-called halfcounterpoise approach, computed as the average of BSSE corrected and uncorrected energy, was recommended for MP2 calculations with small (lower than quadruple- $\zeta$ quality) instead of a full BSSE corrections ${ }^{39}$.

For purposes of comparison with literature data, calculations were also carried out using the large Def2-TZVPP or nearly complete Def2-QZVP basis sets ${ }^{36}$.

The structures of the $\mathrm{C}_{60}$ dimer were taken from reference 28 , while the structures of the C60ISO and iso-C60 datasets are those reported in reference 29 and computed at PBE-D3Def2-TZVPP level of theory. For the sake of clarity, they are sketched in Figure 1.

All calculations were performed with the Gaussian code ${ }^{40}$.

\section{Results and discussion}

\section{$3.1 C_{60}$ dimer}

The first system considered in this study is the weakly bound $\mathrm{C}_{60}$ dimer for which accurate calculations have been recently reported ${ }^{28}$. As mentioned by Clark and collaborators ${ }^{28}$, the interaction between the two molecules in this dimer is largely dominated by dispersion interactions, but, in contrast with other systems, such as those collected in the $\mathrm{L}^{27}$ dataset, the many-body interactions are negligible. This dimer is, therefore, a perfect playground to verify the quality of the proposed PBE-QIDH/DH-SVP model where two-body interactions should be improved with respect to other density functionals by the presence of a PT2 contribution. 
In Table 1 are reported the binding energies for the $\mathrm{C}_{60}$ dimer, computed at different DFT levels using the same reference structure ${ }^{28}$, and completed with reference values obtained with wave-function correlated methods (all these latter values are taken from reference 28). These reference values are relatively homogeneous, ranging between $-11.97(\operatorname{CCSD}(\mathrm{T}))$ and -12.94 (CEPA-1) $\mathrm{kcal} / \mathrm{mol}^{28}$. As expected, the interaction energy computed at the MP2 level is largely overestimated $(-23.46 \mathrm{kcal} / \mathrm{mol})$, whereas the SOS-MP2 result is in better agreement with the reference value $(-14.17 \mathrm{kcal} / \mathrm{mol})$. Concerning DFT approaches, it clearly appears from the data collected in Table 1 , that all the methods missing an empirical potential significantly underestimate the interaction energy. The obtained values range between 6.14 $\mathrm{kcal} / \mathrm{mol}$ at the B3LYP level, i.e. corresponding to an unbound dimer, to $-3.95 \mathrm{kcal} / \mathrm{mol}$ for the B2-PLYP functional, where the negative value well underlines the role of the dynamical correlation contribution.

The empirical corrections have, as expected, a beneficial effect on the binding energies leading in all reported cases to a bound dimer, albeit the estimated energies are roughly one half of the reference values (all between -6 and $-7 \mathrm{kcal} / \mathrm{mol}$ ). The only exceptions are represented by M06-2X-D3(BJ) (-9.7 kcal/mol) and B2-PLYP-D3(BJ) (-11.07 kcal/mol), even if the discrepancy with respect to the reference values is still large. In all these cases (with and without dispersion corrections), these values have been obtained using the Def2TZVP basis set.

In this context, the PBE-QIDH functional coupled with the Def2-TZVP and the D3(BJ) dispersion provides a better agreement with respect to the reference values, its estimation being $-12.29 \mathrm{kcal} / \mathrm{mol}$. A very close result, $-12.27 \mathrm{kcal} / \mathrm{mol}$, is then found using the nonempirical PBE-QIDH/DH-SVPD protocol. Noteworthy, this small basis leads also to significant improvements for two other DHs, namely B2-PLYP (-8.00 kcal/mol) and DSDPBEP86 (-17.40 kcal/mol), thus further proving its transferability.

It should be also remarked that the Def2-QZVP basis has 3720 functions (6120 primitives) for the $\mathrm{C}_{60}$ dimer, while in the case of the smaller DH-SVPD basis only 1200 functions (1920 primitives) are present. Consequently, single point PBE-QIDH calculations with the latter basis set are roughly 4 times faster than those performed with the larger basis (for the $\mathrm{C}_{60}$ dimer at the equilibrium distance) if all other technical factors remain the same.

Beside the interaction energy at a fixed geometry, it is also interesting to analyze the full potential energy profile along the distance between the centers of mass of the two moieties constituting the $\mathrm{C}_{60}$ dimer. This test is even more stringent since the dispersion corrections could fail to reproduce the energy profile far from energy minima ${ }^{41}$, sometime giving spurious 
discontinuities $^{42}$. In our case, however, the validity of this test should be modulated, since the available reference values are calculated at the CEPA-1/cc-pVDZ level, while larger basis set are normally used to reach energies at convergence with this level of theory (triple- or quadruple- $\zeta$, see for instance reference 43 and 44). Indeed, the dissociation energy at the CEPA-1 level is $-12.94 \mathrm{kcal} / \mathrm{mol}$ with the cc-pVTZ basis set, and $-6.88 \mathrm{kcal} / \mathrm{mol}$ with the smaller cc-pVDZ basis set $^{28}$.

The profile computed at the PBE-QIDH/DH-SVPD level is reported in Figure 2 together with other results taken from reference 28 . This profile provides the deepest well with respect to the other ones reported, the reference CEPA-1 being the shallowest one. However, the PBEQIDH/DH-SVPD potential does not show any unphysical discontinuities, as it is also the case for the others approaches tested, and, more interesting, its minimum is shifted toward a shorter distance $(9.75 \AA)$ in slight better agreement with the CEPA-1 results than B3LYPD3(BJ) or VV10-SCNL (9.95 and $9.85 \AA$, respectively). Equilibrium distances and binding energies are also reported in Table 2 for sake of clearness.

In order to give an estimate of the correlation between BSIE and BSSE, this latter was evaluated using different basis sets. Indeed, the BSSE for the DH-SVPD $(5.9 \mathrm{kcal} / \mathrm{mol})$ is, as expected, intermediate between that computed for the smaller Def2-SVPD $(9.8 \mathrm{kcal} / \mathrm{mol})$ and that for the larger Def2-TZVP basis set $(1.7 \mathrm{kcal} / \mathrm{mol})$. In other words, the optimized exponents in the DH-SVPD are the results of the balance between the underestimation of the interaction energy due to BSIE set and its overestimation coming from BSSE.

\subsection{C60ISO and iso-C60 benchmarks}

The second test concerns the relative stability of selected isomers of the $\mathrm{C}_{60}$. Here two different sets have been considered. The first is the so-called C60ISO, composed by the five energetically lowest and by the five energetically highest isomers of $\mathrm{C}_{60}$ selected among the 1812 ones identified by Grimme and co-workers ${ }^{29}$. The second set, named iso-C60, contains 8 isomers chosen so that their relative energies cover a large interval (from 73.5 to 256.7 $\mathrm{kcal} / \mathrm{mol})^{30}$. In the first case, reference energies are computed at $\operatorname{CCSD}(\mathrm{T}) / \mathrm{CBS}$ level, while in the second set reference values are given using a composite method, namely the G4(MP2) approach. In view of their accuracy and the high energy differences, the reference values obtained by these two methods can be considered of a comparable precision.

Results obtained for the C60ISO dataset are reported in Table 3. As it can be easily seen from the data obtained with the Def2-QZVP basis, results obtained adding the empirical dispersion correction (here D3(BJ)) are very similar to those not including it. In particular, a slight 
deterioration of the MADs is observed for most of the functionals, including B3LYP $(+0.22$ $\mathrm{kcal} / \mathrm{mol}), \operatorname{PBE} 0(+0.19 \mathrm{kcal} / \mathrm{mol})$ and PBE0-DH $(+0.21 \mathrm{kcal} / \mathrm{mol})$. An opposite behavior, but always with marginal variations, is found for B2-PLYP $(-0.26 \mathrm{kcal} / \mathrm{mol})$ while PBEQIDH is practically constant $(-0.01 \mathrm{kcal} / \mathrm{mol})$. This trend, already commented in literature ${ }^{27}$, indicates that dispersion interactions play a negligible role in such systems.

It is not therefore surprising that the results obtained with the DH-SVPD basis are also very close. Indeed, these results globally show the same trend observed when the empirical correction is added to the functionals using the larger Def2-QZVP basis. Some exceptions can however be noticed. For instance, the mPW2-PLYP functional is not affected by the D3(BJ) correction when the Def2-QZVP basis set is used (variations of $-0.01 \mathrm{kcal} / \mathrm{mol}$ upon adding it), while the small basis set significantly increases the MAD with respect to the value obtained with the larger basis set $(5.10 \mathrm{vs.} 4.66 \mathrm{kcal} / \mathrm{mol}$, i.e. $+0.44 \mathrm{kcal} / \mathrm{mol})$.

More in general it should be remarked that, despite the apparent high deviations obtained for some functionals, all the functionals provide quite accurate results. Indeed, considering the percent error with respect to the sum of all the total energies, the deviations range between 0.2 $\%$ (B3LYP/Def2-QZVP) and $0.8 \%$ (B2-PLYP/DH-SVPD). Therefore, the overall error is clearly less dramatic than that appears at a first sight.

The same behavior is observed for the other set, iso-C60. Here the MADs computed using the Def2-TZVPP basis set are significantly larger, as it could expected in view of how the isomers have been selected. Indeed, they range between 3.3 (mPW2-PLYP) and 13.9 (PBE0) $\mathrm{kcal} / \mathrm{mol}$. A small decrease of the error is observed when the DH-SVPD basis set is considered, with the PBE-QIDH and mPW2-PLYP functionals providing the lowest MADs, of $3.8 \mathrm{kcal} / \mathrm{mol}$ and $1.5 \mathrm{kcal} / \mathrm{mol}$, values which correspond to a deviation of less than $0.4 \%$ with respect to the sum of all the energies. Such a small deviation is close to those found for the C60ISO set and can be, in our opinion, considered as acceptable for any chemical applications involving this kind of challenging systems.

Despite the fact that the relative stabilities of the C60 isomers for both sets seem not to be ruled by dispersion interactions, these results show the interest in the developed computational protocol. Indeed, in both datasets, the results obtained with the DH-SVPD basis set are very close to (or better) than those obtained with the larger Def2-QZVP or Def2TZVPP basis coupled to empirical corrections showing that this basis set do not deteriorate the description of covalent interactions. The DH-SVPD basis is therefore a valid and fast alterative to more time-consuming protocols. 
It is also interesting to remark that there is not a systematic improvement in going from GGA to hybrids to double hybrids for the relative stabilities of the C60 isomers. Indeed, for the C60ISO set the PBE/Def2-QZVP deviation is $11.06 \mathrm{kcal} / \mathrm{mol}^{30}$, a very low value is found for PBE0 $(2.16 \mathrm{kcal} / \mathrm{mol})$ and finally the PBE-QIDH functional gives a slightly high error (3.48 $\mathrm{kcal} / \mathrm{mol}$ ). A similar trend can be found for the BLYP family. However, the behavior is different for the two sets. Indeed, for the iso-C60 set the MAD increases in going from GGA to $\mathrm{GH}$ and then decreases back when DHs are considered (3.0, 13.9 and $5.5 \mathrm{kcal} / \mathrm{mol}$ for the PBE, PBE0 and PBE-QIDH functionals, respectively, with the Def2-TZVPP basis set). Again, a similar behavior is found with the corresponding functional casting the BLYP model (BLYP, B3LYP and B2-PLYP). At the best of our knowledge ${ }^{45}$, these are one of the very few sets for which there is not a systematic improvement in going from GGA to GH to DH.

It has been argued for the iso-C60 set that the oscillating behavior for MAD is related to the quantity of HF (or exact) exchange introduced in the functional, more than 3\%-5\% deteriorating the results ${ }^{30}$. This argument is, however, not fully applicable to the C60ISO set, where the introduction of a not negligible quantity of HF exchange $(\geq 20 \%)$ significantly improves the results. It is however true that a larger quantity of HF exchange significantly deteriorates the performance. Indeed, the MAD computed for the PBE-QIDH functional without taking into account the PT2 contribution (the so called PBE-QIH model) is 42.5 $\mathrm{kcal} / \mathrm{mol}$ for the iso-C60 set and using the DH-SVPD basis set. This well illustrate the increasing in going from PBE0 (11.9 kcal/mol) to PBE-QIH $(42.5 \mathrm{kcal} / \mathrm{mol})$, which parallels their HF contribution, 25 and $\sim 70 \%$, respectively. This trend is also found for the C60ISO, where the values obtained with the DH-SVPD basis set are 2.2 and $18.3 \mathrm{kcal} / \mathrm{mol}$ for PBE0 and PBE-QIH, respectively. However, in DHs the observed error results from a strong compensation between the large HF contribution and the PT2 counterpart. For instance, the MAD for the iso-C60 set is $42.5 \mathrm{kcal} / \mathrm{mol}$ for PBE-QIH and only $3.8 \mathrm{kcal} / \mathrm{mol}$ for PBE-QIDH (DH-SVPD basis set). A similar behavior can be found for the other DHs when the PT2 contribution is not considered (see Table 3 and 4). As a consequence, the different order found for the computed MADs, GGA > GH < DH for the C60ISO or GGA > GH > DH for iso-C60 depends on how large is the error at GGA level for the given set, an error that could not be efficiently compensated by the PT2 contribution in the final DH. .

\section{Conclusions}

Fullerene represents a challenging and interesting molecule to test new DFT-based approaches: in the tackled dimer, the intermolecular interaction is ruled by weak noncovalent 
forces, which, instead, do not affect the relative stabilities of its isomers, for which an accurate description of the electronic structure is fundamental. On such difficult playground we have tested how double-hybrid functionals coupled to a small basis set tailored for weak interactions (the so-called DH-SVPD basis) may work. Beyond of a detailed comparison with reference values obtained with accurate wave-function methods, we have used as relevant feature the deviations obtained with respect to functionals incorporating empirical dispersion potentials, since these latter represent the most-common approach in the field. Indeed, the DH-SVPD basis set has been specifically developed to avoid their use with double hybrids, without loss in accuracy.

The obtained results confirm that the DH-SVPD values are always very close to those obtained with empirical corrections. Indeed, the PBE-QIDH/DH-SVPD model provides an accuracy for the interaction of the $\mathrm{C}_{60}$ dimer comparable to those obtained with the best functionals using empirical dispersion and larger quadruple- $\zeta$ basis. In contrast, both approaches (tailored basis set and larger basis with empirical potential) do not reach the same level of accuracy in the prediction of relative stabilities of $\mathrm{C}_{60}$ isomers. Nevertheless, this test well underlined how the DH-SVPD basis set can efficiently replace the use of dispersion potentials coupled with larger basis set, at a fraction of the computational cost, thus significantly reducing the computational effort for double hybrids and enabling the description of larger molecular systems.

\section{Acknowledgement}

We thank the authors of references 28,29 and 30 for proving the row data of their results in the Supplementary Information of the articles.

This work has received funding from the European Research Council (ERC) under the European Union's Horizon 2020 research and innovation programme (grant agreement No 648558). E.B. gratefully acknowledges the GENCI-CINES for HPC resources (Projects AP010810360 and A0040810359). ANR (Agence Nationale de la Recherche) and CGI (Commissariat à l'Investissement d'Avenir) are gratefully acknowledged for their financial support of this work through Labex SEAM (Science and Engineering for Advanced Materials and devices) ANR 11 LABX 086, ANR 11 IDEX 0502.

\section{Supporting information}

The Supporting Information is available free of charge on the ACS Publications website: raw data of Table 3 and 4 and Figure 1 obtained with the DH-SVPD basis set. 


\section{References}

1) Sancho-García, J. C.; Adamo, C. Double-hybrid density functionals: merging wavefunction and density approaches to get the best of both worlds. Phys. Chem. Chem. Phys. 2013, 15, 14581-14594.

2) Görling, A, Levy, M. Exact Kohn-Sham scheme based on perturbation theory Phys Rev A 1994, 50, 196-204.

3) Becke, A. D. A new mixing of Hartree-Fock and local density-functional theory. J. Chem. Phys. 1993, 98, 1372-1377.

4) Zhao, Y.; Lynch, B. J.; Truhlar, D. G. Doubly hybrid meta DFT: new multi-coefficient correlation and density functional methods for thermochemistry and thermochemical kinetics J. Phys. Chem. A 2004, 108, 4786-4791.

5) Grimme, S. Semiempirical hybrid density functional with perturbative second-order correlation. J. Chem. Phys. 2006, 124, 034108

6) Goerigk, L.; Grimme, S. A thorough benchmark of density functional methods for general main group thermochemistry, kinetics, and noncovalent interactions Phys. Chem. Chem. Phys., 2011,13, 6670-6688

7) Calbo, J.; Ortí, E. ; Sancho-García, J.C.; Aragó, J. Accurate treatment of large supramolecular complexes by double-hybrid density functionals coupled with nonlocal van der Waals corrections. J. Chem. Theory Comput. 2015, 11, 932-939

8) Sancho-García, J.C; Brémond, E.; Savarese, M.; Pérez-Jiménez, A. J.; Adamo, C. Partnering dispersion corrections with modern parameter-free double-hybrid density functionals Phys. Chem. Chem. Phys. 2017, 19, 13481-13487.

9) Pérez-Jiménez , A.J.; Brémond, E.; Adamo, C; Sancho-Garcia, J.C.; Adamo, C; Accurate description of interaction energies and three-body effects in weakly bound molecular complexes by PBE-QIDH models. J. Chem. Phys. 2018, 149, 041101

10) $\mathrm{Wu}, \mathrm{Q}$; Y Yang, W. Empirical correction to density functional theory for van der Waals interactions J. Chem. Phys. 2002, 116, 515-524.

11) Grimme, S. Accurate description of van der Waals complexes by density functional theory including empirical corrections J. Comput. Chem. 2004, 25, 1463-1473.

12) Risthaus, T.; Grimme, S. Benchmarking of London dispersion-accounting density functional theory methods on very large molecular complexes, J. Chem. Theory Comp. 2013, 9, 1580-1591. 
13) Grimme, S.; Antony, J.; Ehrlich, S.; Krieg, H. A consistent and accurate ab initio parametrization of density functional dispersion correction (DFT-D) for the 94 elements HPu. J. Chem. Phys. 2010, 132, 154104.

14) Grimme, S.; Ehrlich, S.; Goerigk, L. Effect of the damping function in dispersion corrected density functional theory J. Comp. Chem. 2011, 32, 1456-1465.

15) von Lilienfeld, O.A.; Tkatchenko, A. Two- and three-body interatomic dispersion energy contributions to binding in molecules and solids. J. Chem. Phys. 2010, 132, 234109.

16) Brauer, B.; Kesharwani, M.K.; Kozuch, S.; Martin, J.M.L. The S66x8 benchmark for noncovalent interactions revisited: explicitly correlated ab initio methods and density functional theory Phys. Chem. Chem. Phys. 2016, 18, 20905-20925.

17) Brémond, É.; Sancho-García, J. C.; Pérez-Jiménez, Á. J.; Adamo, C. Double-hybrid functionals from adiabatic-connection: the PBE-QIDH model. J. Chem. Phys. 2014, 141, 031101-031104

18) Becke, A. D. Density-functional thermochemistry. III. The role of exact exchange. $J$. Chem. Phys. 1993, 98, 5648-5652.

19) Cohen, A. J.; Mori-Sanchez, P.; Yang, W. Assessment and formal properties of exchangecorrelation functionals constructed from the adiabatic connection. J. Chem. Phys. 2007, 127, 034101

20) Toulouse, J.; Sharkas, K.; Brémond, E.; Adamo, C. Rationale for a new class of doublehybrid approximations in density-functional theory. J. Chem. Phys. 2011, 135, 101102.

21) Brémond, E.; Ciofini,I.; Sancho-Garcia, J.C.; Adamo, C. Nonempirical double-hybrid functionals: An effective tool for chemists. Acc. Chem. Res. 2016, 49, 1503-1513.

22) Tsuneda, T.; Suzumura, T.; Hirao, K. A reexamination of exchange energy functionals $J$. Chem. Phys. 1999, 111, 5656-5667

23) Sanz García, J.; Brémond, E. ; Campetella, M. ; Ciofini, I.; Adamo, C. Small basis set allowing the recovery of dispersion interactions with double-hybrid functionals, J. Chem. Theory Comp. 2019, 15, 2944-2953.

24) Varandas, A.J.C., Zeroth-order exchange energy as a criterion for optimized atomic basis sets in interatomic force calculations. Application to $\mathrm{He}_{2}$ Chem. Phys. Lett. 1980, 69, 222-224 25) Gráfová,L.; Pitoňák, M.; Řezáč, J.; Hobza, P. Comparative study of selected wave Function and density functional methods for noncovalent interaction energy calculations using the extended S22 data set, J. Chem. Theory Comp., 2010, 6, 2365-2376

26) Řezáč, J.; Riley, K.E.; Hobza, P. S66: A well-balanced database of benchmark interaction energies relevant to biomolecular structures J. Chem. Theory Comp., 2011, 7, 
2427-2438.

27) Sedlak, R.; Janowski, T.; Pitoňák, M.; Řezáč, J.; Pulay, P.; Hobza, P. Accuracy of quantum chemical methods for large noncovalent complexes J. Chem. Theory Comp. 2013, 9, 3364-3374.

28) Sharapa, D.J.; Margraf, J.T.; Hesselmann, A. Clark, T. Accurate intermolecular potential for the C60 dimer: the performance of different levels of quantum theory, J. Chem. Theory Comput. 2017, 13, 274-285.

29) Sure, R.; Hansen, A.; Schwerdtfegerb, P.; Grimme, S. Comprehensive theoretical study of all 1812 C60 isomers Phys.Chem.Chem.Phys. 2017, 19, 14296-14305

30) Karton, A.; Waite, S.L. ; Page, A.J. . Performance of DFT for C60 isomerization energies: a noticeable exception to Jacob's ladder J. Phys. Chem. A 2019, 123, 257-266

31) Brémond, É.; Adamo, C. Seeking for parameter-free double hybrid functionals: the PBE0DH model. J. Chem. Phys. 2011, 135, 024106.

32) Kozuch, S.; Martin, J.M.L. DSD-PBEP86: in search of the best double-hybrid DFT with spin-component scaled MP2 and dispersion corrections, Phys. Chem. Chem. Phys., 2011, 13, 20104-20107.

33) Becke, A. D.; Johnson, E. R. Exchange-hole dipole moment and the dispersion interaction revisited. J. Chem. Phys. 2007, 127, 154108.

34) Bousquet, D; Brémond, E; Sancho-Garcia; J.C.; Ciofini, I.; Adamo, C. Non-parametrized functionals with empirical dispersion corrections: A happy match? Theor. Chem. Acc. 2015, 134, 1602.

35) Sancho-Garcia, J.C.; Brémond, E.; Savarese, M.; Pérez-Jiménez, A.J.; Adamo, C. Partnering dispersion corrections with modern parameter-free double-hybrid density functionals, Phys. Chem. Chem. Phys. 2017, 19, 13481.

36) Weigend, F.; Ahlrichs, R. Balanced basis sets of split valence, triple zeta valence and quadruple zeta valence quality for $\mathrm{H}$ to $\mathrm{Rn}$ : design and assessment of accuracy. Phys. Chem. Chem. Phys. 2005, 7, 3297-3305.

37) Sheng, X.W.; Mentel, L.; Gritsenko, O.V.; Baerends, E.J. Counterpoise correction is not useful for short and van der Waals distances but may be useful at long range? J. Comp. Chem. 2011, 32, 2896-2901.

38) Alvarez-Idaboy, J.R.; Galano, A.; Counterpoise corrected interaction energies are not systematically better than uncorrected ones: comparison with $\operatorname{CCSD}(\mathrm{T}) \operatorname{CBS}$ extrapolated values Theor. Chem. Acc. 2010, 126, 75-85 
39) Burns, L.A.; Marshall, M.S.; Sherrill, C.D. Comparing counterpoise-corrected, uncorrected, and averaged binding energies for benchmarking noncovalent interactions $J$. Chem. Theory Comput. 2014, 10, 49-57.

40) Gaussian 16, Revision B.01, Frisch, M. J.; Trucks, G. W.; Schlegel, H. B.; Scuseria, G. E.; Robb, M. A.; Cheeseman, J. R.; Scalmani, G.; Barone, V.; Petersson, G. A.; Nakatsuji, H.; Li, X.; Caricato, M.; Marenich, A. V.; Bloino, J.; Janesko, B. G.; Gomperts, R.; Mennucci, B.; Hratchian, H. P.; Ortiz, J. V.; Izmaylov, A. F.; Sonnenberg, J. L.; Williams-Young, D.; Ding, F.; Lipparini, F.; Egidi, F.; Goings, J.; Peng, B.; Petrone, A.; Henderson, T.; Ranasinghe, D.; Zakrzewski, V. G.; Gao, J.; Rega, N.; Zheng, G.; Liang, W.; Hada, M.; Ehara, M.; Toyota, K.; Fukuda, R.; Hasegawa, J.; Ishida, M.; Nakajima, T.; Honda, Y.; Kitao, O.; Nakai, H.; Vreven, T.; Throssell, K.; Montgomery, J. A., Jr.; Peralta, J. E.; Ogliaro, F.; Bearpark, M. J.; Heyd, J. J.; Brothers, E. N.; Kudin, K. N.; Staroverov, V. N.; Keith, T. A.; Kobayashi, R.; Normand, J.; Raghavachari, K.; Rendell, A. P.; Burant, J. C.; Iyengar, S. S.; Tomasi, J.; Cossi, M.; Millam, J. M.; Klene, M.; Adamo, C.; Cammi, R.; Ochterski, J. W.; Martin, R. L.; Morokuma, K.; Farkas, O.; Foresman, J. B.; Fox, D. J. Gaussian, Inc., Wallingford CT, 2016.

41) Gould, T.; Johnson, E.R.; Tawfik, S.A. Are dispersion corrections accurate outside equilibrium? A case study of benzene. Beilstein J. Org. Chem. 2018, 14, 1181-1191.

42) Savarese, M.; Brémond, E.; Adamo, C.; Exploring the limits of recent exchangecorrelation functionals in modeling lithium/benzene interaction, Theor. Chem. Acc. 2016, 135, 99.

43) Di. G. Liakos, A. Hansen, F.Neese Weak molecular interactions studied with parallel implementations of the local pair natural orbital coupled pair and coupled cluster methods $J$. Chem. Theory Comput. 2011, 7, 76-87.

44) F. Neese, A. Hansen, F. Wennmohs, S. Grimme, Accurate theoretical chemistry with coupled pair models, Acc. Chem. Res., 2009, 42, 641-648.

45) Brémond, E.; Savarese, M.; Pérez-Jiménez, A. J. Sancho-García, J.C; Adamo, C. Systematic improvement of density functionals through parameter-free hybridization schemes, J. Phys. Chem. Lett. 2015, 6, 3540-3545 
Table 1. Binding energies $(\Delta \mathrm{E}, \mathrm{kcal} / \mathrm{mol})$ for the $\mathrm{C}_{60}$ dimer.

\begin{tabular}{ccc}
\hline Method & Basis set & $\Delta \mathrm{E}$ \\
\hline DLPNO-CCSD $^{\mathrm{a}}$ & CBS & -12.15 \\
DLPNO-CCSD(T) $^{\mathrm{a}}$ & CBS & -11.97 \\
DLPNO-CEPA-1 $^{\mathrm{a}}$ & CBS & -12.94 \\
MP2 $^{\mathrm{a}}$ & Def2-TZVP & -23.46 \\
SOS-MP2 $^{\mathrm{a}}$ & Def2-TZVP & -14.17 \\
B2-PLYP $^{\mathrm{a}}$ & Def2-TZVP & -3.95 \\
B2-PLYP-D3(BJ) $^{\text {a }}$ & Def2-TZVP & -11.07 \\
DSD-PBEP86-NL $^{\mathrm{a}}$ & Def2-TZVP & -0.19 \\
B3LYP $^{\mathrm{a}}$ & Def2-TZVP & 6.14 \\
B3LYP-NL $^{\mathrm{a}}$ & Def2-TZVP & -7.81 \\
B3LYP-D3(BJ) $^{\mathrm{a}}$ & Def2-TZVP & -7.09 \\
WB97X-D3 $^{\mathrm{a}}$ & Def2-TZVP & -6.03 \\
M06-2X $^{\mathrm{a}}$ & Def2-TZVP & -2.64 \\
M06-2X-D3(BJ) $^{\text {a }}$ & Def2-TZVP & -9.69 \\
PBE-QIDH-D3(BJ) & Def2-TZVP & -12.29 \\
B2-PLYP & DH-SVPD & -8.00 \\
DSD-PBEP86 & DH-SVPD & -17.40 \\
PBE-QIDH & DH-SVPD & -12.27
\end{tabular}

a) From reference 28 
Table 2. Equilibrium distances $(\mathrm{Re}, \AA)$ and binding energies $(\Delta \mathrm{E}, \mathrm{kcal} / \mathrm{mol})$ of the $\mathrm{C}_{60}$ dimer, extracted from the energy profiles reported in Figure 1.

\begin{tabular}{cccc}
\hline Method & Basis set & $\mathrm{R}_{\mathrm{e}}$ & $\Delta \mathrm{E}$ \\
\hline DLPNO-CEPA-1 $^{\text {a }}$ & cc-pVDZ & 9.75 & -6.88 \\
B3LYP-D3(BJ) $^{\text {a }}$ & cc-pVDZ & 9.85 & -7.73 \\
VV-SCNL $^{\mathrm{a}}$ & cc-pVDZ & 9.85 & -9.27 \\
BP86-D3(BJ) $^{\text {a }}$ & Def2-TZVP & 9.75 & -10.07 \\
PBE-QIDH & DH-SVPD & 9.75 & -12.27
\end{tabular}

a) From reference 28 
Table 3. Mean Absolute Deviations (kcal/mol) for the C60ISO set by Grimme. Reference values are computed at the DLPNO-CCSD(T)/CBS level ${ }^{29}$. In parenthesis are reported the values obtained without inclusion of the PT2 contribution to the exchange-correlation energy.

\section{Def2-QZVP Def2-QZVP+D3(BJ)}

\section{DH-SVPD}

$\begin{array}{cccc}\text { B3LYP } & 2.01 & 2.22 & 2.27 \\ \text { PBE0 } & 2.16 & 2.35 & 2.21 \\ \text { PBE0-DH } & 2.91 & 3.12 & 2.84 \\ \text { B2-PLYP } & 7.02 & 6.76 & 7.49(12.72) \\ \text { mPW2-PLYP } & 4.66 & 4.65 & 5.10(13.49) \\ \text { DSD-PBEP86 } & 7.86 & 7.56 & 7.47 \\ \text { PBE-QIDH } & 3.48 & 3.47 & 4.26(18.32)\end{array}$


Table 4. Mean Absolute Deviations (kcal/mol) for the iso-C60 set by Karton et co-workers ${ }^{30}$. Reference values are computed at the G4(MP2) level. In parenthesis are reported the values obtained without inclusion of the PT2 contribution to the exchange-correlation energy.

Def2-TZVPP Def2-TZVPP+D3(BJ) DH-SVPD

$\begin{array}{cccc}\text { B3LYP } & 11.06 & 11.16 & 9.35 \\ \text { PBE0 } & 13.29 & 13.30 & 11.93 \\ \text { PBE0-DH } & 13.70 & 13.85 & 12.18 \\ \text { B2-PLYP } & 3.59 & 3.81 & 4.83(32.68) \\ \text { mPW2-PLYP } & 3.39 & 2.38 & 1.50(34.11) \\ \text { DSD-PBEP86 } & 4.83 & 4.82 & 6.21 \\ \text { PBE-QIDH } & 5.54 & 5.58 & 3.82(42.50)\end{array}$




\section{Figure captions}

Figure 1. Structures of the fullerene isomers in the C60ISO (top) and iso-C60 (bottom) set. References energies (DLPNO-CCSD(T)/CBS for the former and G4(MP2) for the latter) and point group symmetries are also reported (in $\mathrm{kcal} / \mathrm{mol}$ ).

Figure 2. Potential energy profile of the fullerene dimer computed as function of the distance between the $\mathrm{C}_{60}$ centers of mass. CEPA-1, B3LYP-D3(BJ), VV10-SCNL and BP86-D3(BJ) data are taken from reference 28. 


\section{C60ISO}

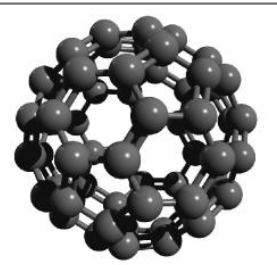

$I_{\mathrm{h}}(0.00)$

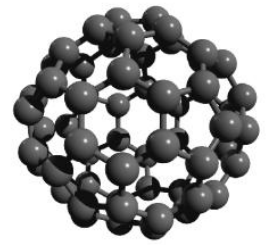

$C_{2}(69.89)$

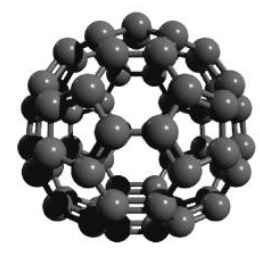

$C_{2 \mathrm{v}}(37.40)$

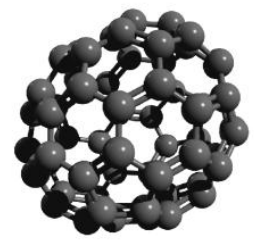

$C_{1}(100.48)$

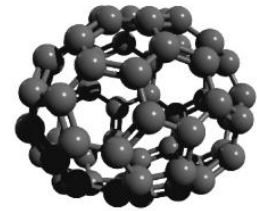

$C_{2 \mathrm{~h}}(143.96)$

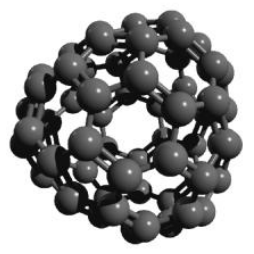

$D_{3}(56.87)$

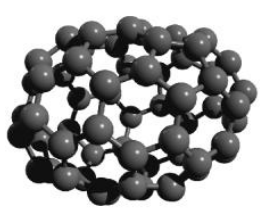

$C_{1}(111.72)$

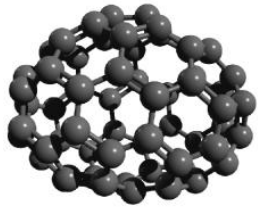

$C_{2}(164.88)$

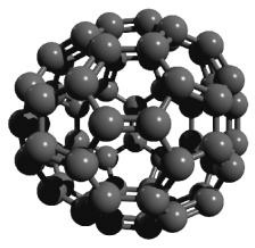

$C_{\mathrm{s}}(56.89)$

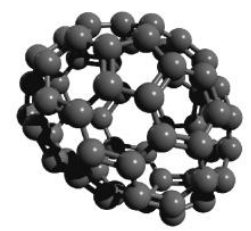

$C_{\mathrm{s}}(142.18)$

\section{iso- $\mathrm{C} 60$}

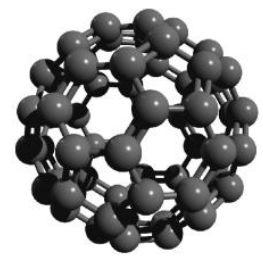

$I_{\mathrm{h}}(0.00)$

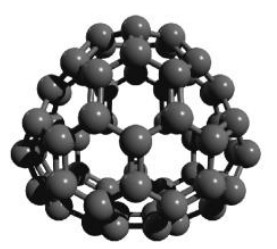

$C_{2 \mathrm{v}}(134.44)$

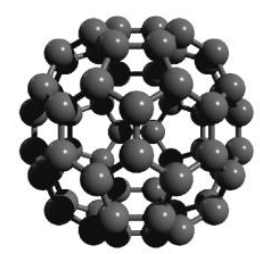

$D_{2 \mathrm{~d}}(73.49)$

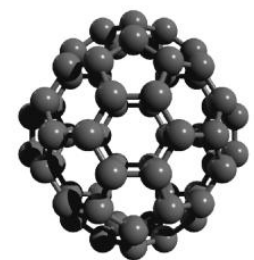

$C_{2 \mathrm{v}}(155.00)$

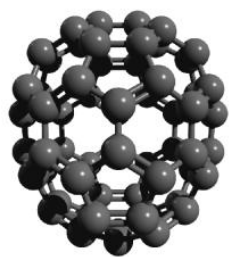

$D_{2 \mathrm{~d}}(74.43)$

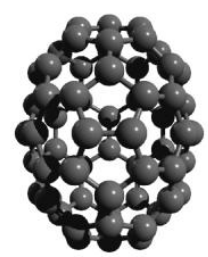

$C_{2 \mathrm{v}}(166.16)$

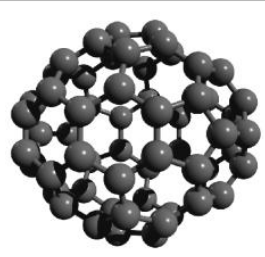

$C_{2 \mathrm{~h}}(133.46)$

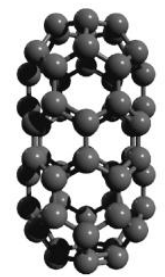

$D_{6 \mathrm{~h}}(256.69)$

Figure 1 


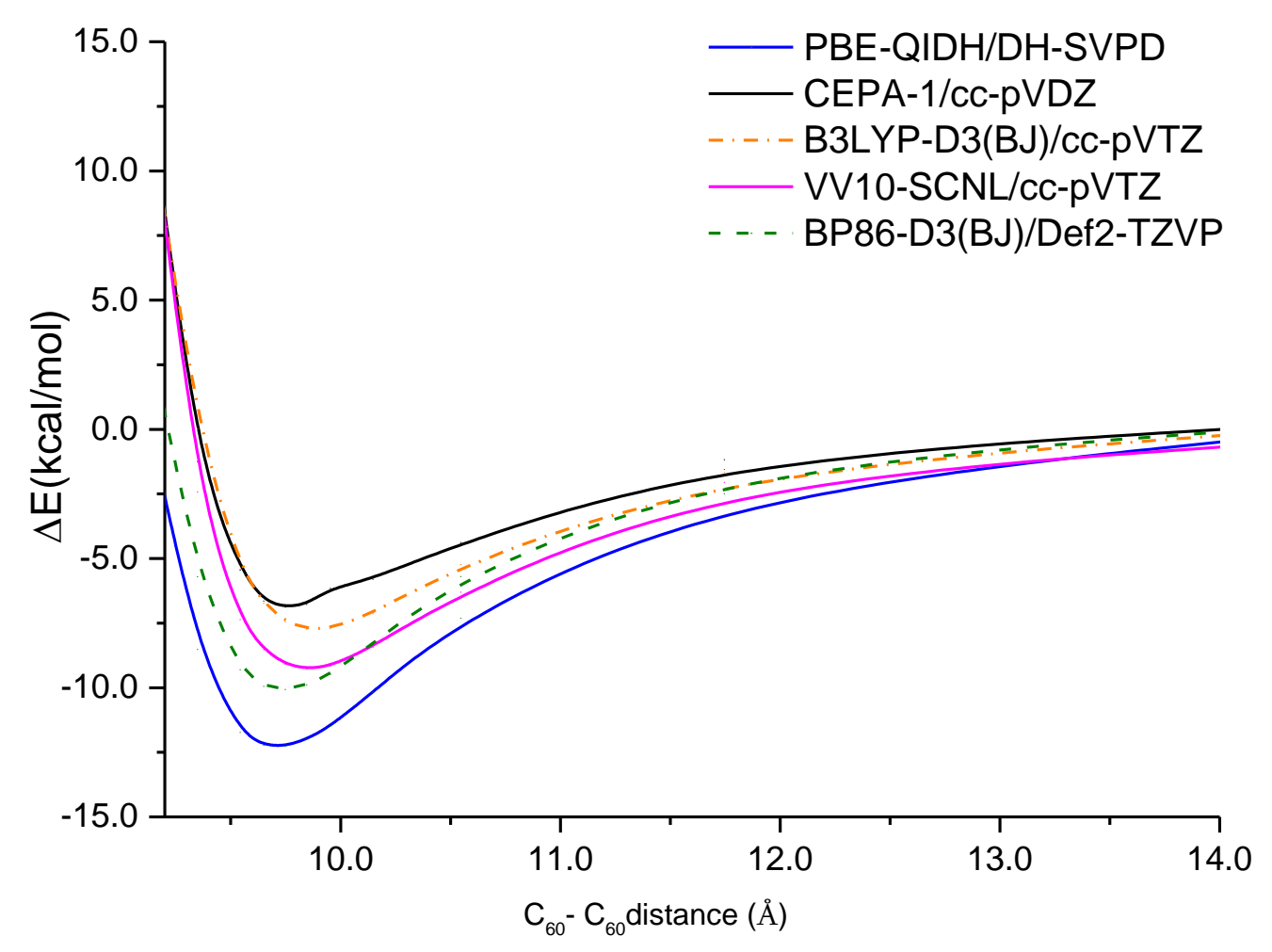

Figure 2 


\section{TOC Graphic}

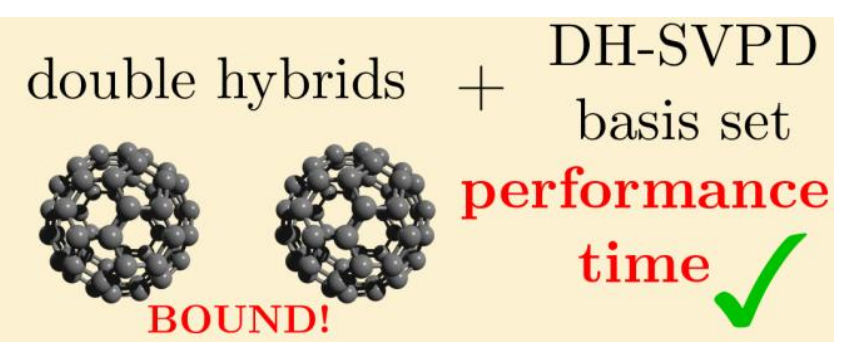

